\title{
ESTIMATION OF ADSORPTION PARAMETERS OF A BINARY SYSTEM BY APPLYING THE LEWIS RULE
}

KANJI MIYABE*

Central Laboratories, Kurita water Industries Ltd., 7-1, Wakamiya, Morinosato, Atsugi 243-01

MOTOYUKI SUZUKI

Institute of Industrial Science, University of Tokyo, 7-22-1 Roppongi, Minato-ku, Tokyo 106

Key Words: $\quad$ Reversed Phase Chromatography, Adsorption Equilibrium, Liquid Phase Adsorption, Binary System, Langmuir Equation, Markham Benton Equation, Lewis Equation

\section{Introduction}

Reversed-phase liquid chromatography has been gaining popularity as one of the most reliable and versatile tools for analytical separation and quantification of mixtures $^{8)}$. In the course of development of this chromatographic technique, considerable attention has been given to its preparative use on a large scale as well as for analytical purposes $1,2,4,5,10,11)$. It is important to develop not only superior preparative equipment that achieves high chromatographic efficiency but also a standard strategy for the scale-up approach to preparative separations.

Preparative chromatography is often operated under mass overload conditions, unlike an analytical procedure $^{3)}$. The development of preparative separations is accompanied by additional problems such as non-linearity of an adsorption isotherm and the concentration dependence of diffusivity. Because the properties of preparative separations differ considerably from those of analytical ones, conventional theory for analytical liquid chromatography cannot be applied directly to preparative separations. Simulation of chromatographic processes is useful in establishing a strategy for optimization of preparative operations ${ }^{5)}$. First, exact representation of adsorption equilibria is required to simulate chromatographic separations accurately.

A number of methods based on the combination of single-component isotherms have been proposed for estimation of adsorption isotherms for multicomponent systems $^{9}$. In the previous paper, the authors reported a simple method for estimation of an adsorption isotherm by analyzing a desorption curve ${ }^{6)}$. This paper is concerned with adsorption equilibria of binary systems in reversed-phase liquid chromatography.

\section{Experimental}

A high-performance liquid chromatograph equipment unit was employed. A small volume of sample

\footnotetext{
* Received October 28, 1993. Correspondence concerning this article should be addressed to K. Miyabe.
}

solutions was introduced into a fluid flow by use of a sample injector. A column was kept at $298 \pm 0.1 \mathrm{~K}$ in a thermostated water bath. The concentration of the sample in effluent was monitored by an ultraviolet detector.

The column was $6 \mathrm{~mm}$ in inside diameter and 150 $\mathrm{mm}$ in length. The average diameter of ODS particles was $45 \mu \mathrm{m}$. The carrier solvent was a mixture of methanol and pure water (50/50,vol.). As sample materials, $p$ ethylphenol (PEP), 1-naphthol (1-NAPH), p-secbutylphenol (PSBP), and p-tert-butylphenol (PTBP) were used, with uracil as an inert substance.

Adsorption isotherms of the adsorbates were determined by breakthrough and desorption experiments. The concentration of the adsorbates was changed stepwise from $1 \times 10^{-3}$ to $1 \times 10^{-2} \mathrm{~g} / \mathrm{cm}^{3}$. For binary systems, measurements of adsorption isotherms were carried out in the same manner at various concentrations of each component in the concentration range.

\section{Results and Discussion}

\subsection{Adsorption isotherms of each adsorbate}

Single-component isotherms of the adsorbates could be expressed by the Langmuir equation, as was shown in the previous paper ${ }^{6,7)}$. Langmuir parameters used are listed in Table 1. The figures of saturated amount adsorbed were of the same order of magnitude.

\subsection{Adsorption equilibria of binary systems}

An attempt was made to represent the adsorption isotherms of the binary systems by the Markham-Benton equation ${ }^{9)}$.

$$
q_{i}=q_{m i} K_{i} c_{i} /\left(1+\Sigma K_{i} c_{i}\right)
$$

Adsorbed amounts were calculated by using the Langmuir parameters listed in Table 1 without modification. Examples of the binary system of $p$-ethylphenol and 1naphthol are shown in Figs. 1 and 2. Adsorption equilibria of the binary system could be represented by Eq. (1). Similar results could be obtained for the binary 
Table 1. Langmuir parameters

\begin{tabular}{lcc}
\hline \multicolumn{1}{c}{ Adsorbate } & $q_{m}[\mathrm{mmol} / \mathrm{g}]$ & $K\left[\mathrm{~cm}^{3} / \mathrm{mmol}\right]$ \\
\hline p-Ethylphenol & 0.94 & 8.66 \\
1-Naphthol & 0.75 & 13.6 \\
p-sec-Buthylphenol & 0.70 & 13.8 \\
p-tert-Buthylphenol & 0.70 & 11.4 \\
\hline
\end{tabular}

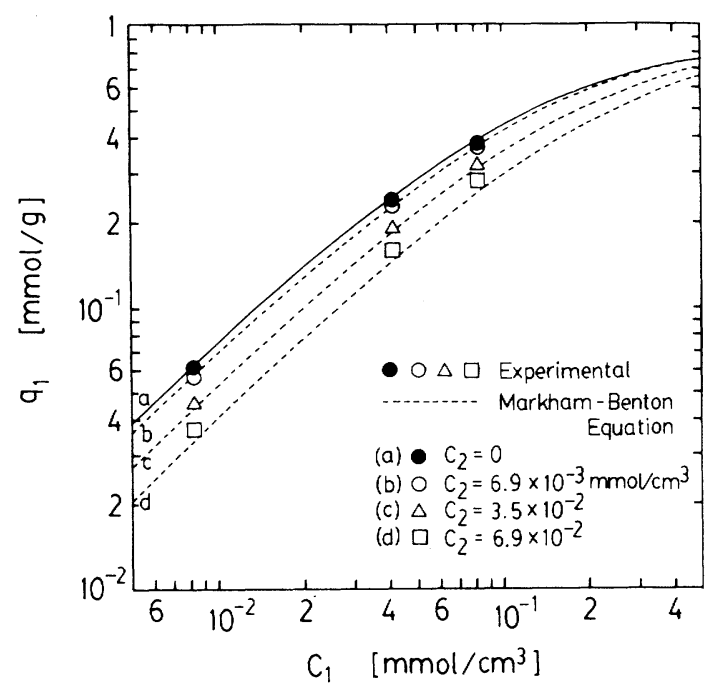

Fig. 1 Adsorption from binary-solute system: p-ethylphenol (1) - 1-naphthol (2) at $298 \mathrm{~K}$

system of $p$-sec-butylphenol and p-tert-butylphenol.

\subsection{Estimation of langmuir parameters}

Adsorption equilibria of binary systems in the reversed-phase mode can be expressed by the MarkhamBenton equation with Langmuir parameters of singlecomponent isotherms. In many cases, the isotherm of an objective component can be determined by using purified substance. An adsorption isotherm of a contaminant, however, cannot be measured directly because its standard material cannot be obtained. It is necessary to develop an estimation method of Langmuir parameters from adsorption data in binary systems by using a small amount of samples since objective components purified by reversed-phase liquid chromatography are usually expensive.

When the applicability of the Markham-Benton equation is established, the Lewis relation is expected to hold $^{9)}$.

$$
q_{i} / q_{i}^{o}+q_{j} / q_{j}^{o}=1
$$

Adsorption data of the binary systems in this study are plotted according to Eq. (2). The value of $q_{i}^{o}$ was calculated with the Langmuir equation of i-th component by assuming that $c_{i}$ was equal to $c^{0}$. Similarly for the other component $j, q_{j}^{0}$ was calculated by using the Langmuir equation of $j$-th component under the condition that $c_{j}$ was equal to $c^{0}$. As shown in Fig. 3, a linear relation was observed. It is confirmed that the Lewis equation holds in a reversed-phase liquid chromatographic system.

The following procedure can be proposed for esti-

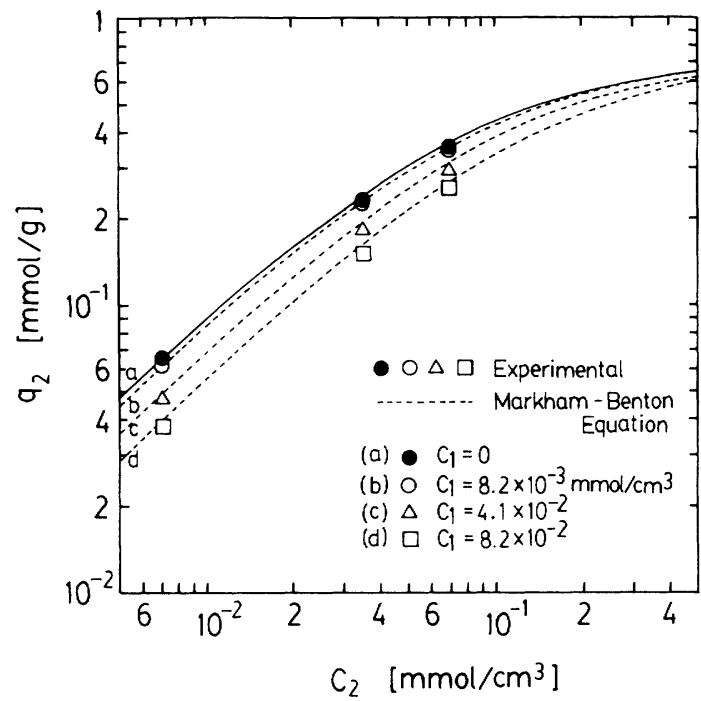

Fig. 2 Adsorption from binary-solute system: p-ethylphenol (1) - 1-naphthol (2) at $298 \mathrm{~K}$

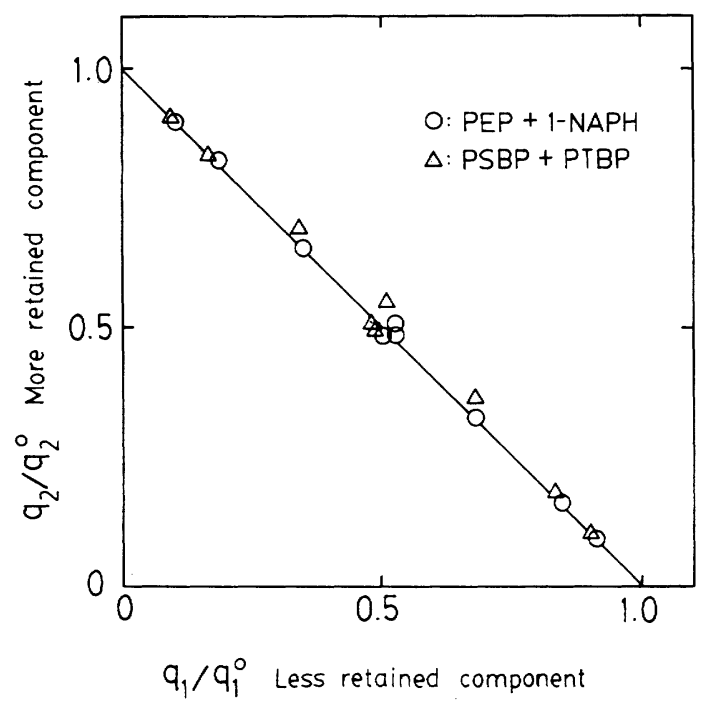

Fig. 3 Lewis plot of amounts adsorbed on ODS

mation of Langmuir parameters from adsorption data of binary systems by applying the Lewis equation. Adsorbability and physical properties of contaminants are assumed to be similar to those of objective components because the contaminants are usually by-products of synthesis or fermentation steps in practice.

(1) Henry constants, $q_{m i} K_{i}$ and $q_{m j} K_{j}$, for an objective component $(i)$ and a contaminant $(j)$ are determined from pulse response experiments.

(2) According to the simple methods proposed in the previous paper ${ }^{6)}$, the saturated amount adsorbed $q_{m i}$ and the adsorption equilibrium constant $K_{i}$ are determined.

(3) The value of $q_{i}^{0}$ in Eq. (2) can be calculated from the total concentration of the components in a binary system because Langmuir parameters $q_{m i}$ and $K_{i}$ of $i$-th component are already known. As the amounts of the components adsorbed in the binary system, $q_{i}$ and $q_{j}$, 
Table 2. Estimation of Langmuir parameters for the binarysolute system of p-Ethylphenol and 1-Naphthol by the Lewis equation

\begin{tabular}{|c|c|c|c|c|c|c|}
\hline \multirow[t]{2}{*}{ Solute } & \multicolumn{2}{|c|}{ Exptl. } & \multicolumn{2}{|c|}{ Calcd. } & \multicolumn{2}{|c|}{ Calcd. } \\
\hline & $\begin{array}{c}q_{m} \\
{[\mathrm{mmol} / \mathrm{g}]}\end{array}$ & $\begin{array}{c}K \\
\mathrm{~m}^{3} / \mathrm{mmol}\end{array}$ & $\begin{array}{c}q_{m} \\
{[\mathrm{mmol} / \mathrm{g}}\end{array}$ & $\begin{array}{c}K \\
{\left[\mathrm{~cm}^{3} / \mathrm{mmol}\right]}\end{array}$ & $\begin{array}{c}q_{m} \\
{[\mathrm{mmol} / \mathrm{g}]}\end{array}$ & $\begin{array}{c}K \\
{\left[\mathrm{~cm}^{3} / \mathrm{mmol}\right]}\end{array}$ \\
\hline PEP & 0.94 & 8.66 & Objective & component & 0.98 & 8.47 \\
\hline 1-NAPH & 0.75 & 13.6 & 0.78 & 13.3 & Objective & component \\
\hline
\end{tabular}

are experimentally measured, $q_{j}^{0}$ can be obtained from Eq. (2). When a single-component isotherm can be represented by the Langmuir equation, $q_{j}^{0}$ can be expressed as follows.

$$
q_{j}^{o}=q_{m j} K_{j} c^{o} /\left(1+K_{j} c^{o}\right)
$$

Because the values of $q_{j}^{0}, q_{m j} K_{j}$, and $c^{0}$ in Eq. (3) are already known, firstly $K_{j}$ can be calculated. Then $q_{m j}$ can be obtained by dividing the Henry constant of $j$-th component, $q_{m j} K_{j}$, by the value of $K_{j}$ calculated previously.

As shown in Table 2, good agreement was observed between Langmuir parameters experimentally determined and the values estimated by the proposed method. In Table 2, Langmuir parameters were calculated for each case, in which $p$-ethylphenol or 1-naphthol was assumed to be an objective component. To obtain accurate results by the proposed method, as many adsorption experiments as possible must be carried out. Changes in composition of components, however, are not required. Changes in the degree of dilution of a sample suffice for the change in adsorption conditions.

Estimation of adsorption isotherms in multicomponent systems has been carried out on the basis of the combination of single-component isotherms ${ }^{9)}$. In this work the characteristics of binary adsorption equilibria in reversed-phase liquid chromatography were studied. It was experimentally confirmed that a binary adsorption isotherm could be expressed by the Markham-Benton equation, using Langmuir parameters for single adsorp- tion isotherms, and that the Lewis relation held for the reversed-phase liquid chromatographic mode. On the basis of the results, a simple method was proposed for the estimation of Langmuir parameters from the adsorption data of a binary system. The method requires in practice only three preliminary experimentally measured data, namely one pulse response curve and one breakthrough curve of a mixture and one desorption curve of an objective component.

\section{Acknowledgement}

The authors thank Mr. S. Sakuma for his experimental work.

\section{Nomenclature}

$c \quad=$ concentration in mobile phase $\quad\left[\mathrm{mmol} / \mathrm{cm}^{3}\right]$

$c^{0}=c_{i}+c_{j}$ total concentration of a binary system,

$$
c_{i}+c_{j} \quad\left[\mathrm{mmol} / \mathrm{cm}^{3}\right]
$$

$K=$ adsorption equilibrium constant $\quad\left[\mathrm{cm}^{3} / \mathrm{g}\right]$

$q \quad=$ amount adsorbed $\quad[\mathrm{mmol} / \mathrm{g}$ ]

$q_{m} \quad=$ saturated amount adsorbed $\quad[\mathrm{mmol} / \mathrm{g}]$

$q^{0} \quad=$ amount adsorbed in equilibrium with $c^{0} \quad[\mathrm{mmol} / \mathrm{g}]$

$<$ Subscripts $>$

$i \quad=i$-th component (objective component)

$j \quad=j$-th component (contaminant)

\section{Literature Cited}

1) Bidlingmeyer, B.A.: "Preparative Liquid Chromatography", p 203-233, Elsevier, Amsterdam, Netherlands (1987)

2) Cantwell, A.M., R. Calderone and M. Sienko: J. Chromatogr., 316, 133-149 (1984)

3) Knox, J.H. and H.M. Pyper: J. Chromatogr., 363, 1-30 (1986)

4) Kroeff, E.P., R.A. Owens, E.L. Campbell, R.D. Johnson and H.I. Marks: J. Chromatogr., 461, 45-61 (1989)

5) Miyabe, K., T. Kawazoe, M. Yamaguchi and K. Kitazawa: Kagaku Kogaku Ronbunshu, 15, 475-480 (1989)

6) Miyabe, K. and M. Suzuki: J. Chem. Eng. Japan, 24, 772-777 (1991)

7) Miyabe, K. and M. Suzuki: AIChE J., 38, $901-910$ (1992)

8) Sander, L.C. and S.A. Wise: CRC Critical Reviews in Analytical Chemistry, 18, 299-415 (1987)

9) Suzuki, M.: “Adsorption Engineering”, p 56-60, Kodansha/ Elsevier, Tokyo, Japan (1990)

10) Verzele, M.: Anal. Chem., 62, 265A-269A (1990)

11) Verzele, M. and E. Geereart: J. Chromatogr.Sci., 18, 559-570 (1980) 